\title{
MAGNETIC HYSTERESIS DYNAMIC MODEL: VARIABLE FREQUENCY PARAMETERS ADJUST.
}

\author{
PEDRO SÉRGIO PEREIRALIMA ${ }^{1}$ \\ 1. Engenharia de instrumentação, Automação e Robótica da UFABC \\ Av dos Estados, 5001 - Santo André (SP) \\ peralima@ufabc.edu.br
}

\begin{abstract}
It is proposed a dynamical model to describe the magnetic hysteresis with two state variables: the intensity magnetic field $H$ and the polarization field $M$; and two non-linear function, the first responsible for the characterization of the anhysteretic curve $f\left(H^{*}\right)$, and the other responsible for the characterization of the Barkhausen noise, $g(M)$. It is made a discussion about the adhesion properties of this model to observed behavior of the hysteretic magnetic curve, it is also presented a comparison between the predictions and the experimental data in four different frequencies $5 \mathrm{mHz}, 50 \mathrm{mHz}, 20 \mathrm{~Hz}$ and $60 \mathrm{~Hz}$.
\end{abstract}

Keywords— magnetic hysteresis, dynamic model.

\section{Introduction}

This work proposes a dynamical approach to describe the magnetic hysteresis. It is a second order model, being the apparent magnetic field $\mathrm{H}$ and the magnetization field $M$ its state variables. Two nonlinear functions $\mathrm{f}($.$) , the an-hysterical magnetization$ curve, and $\mathrm{g}($.$) , the Barkhausen noise, complete the$ model.

A parameter fitness is obtained for different excitation frequencies: $5,50 \mathrm{mHz}$ for $1,5 \mathrm{~T}$ and 20,60 $\mathrm{Hz}$ for 1,7T and 1,5 $\mathrm{T}$ respectively. The data used refer to one single iron-siliceous material used in Epstein Square calibration..

\section{Model Assumptions}

\subsection{Physical Model}

The electromagnetic apparatus is very simple a cooper coil turned around a magnetic nucleus torus feed by an alternating voltage generator, seeing in figure 1a. Its electrical circuit is shown in figure $1 \mathrm{~b}$.

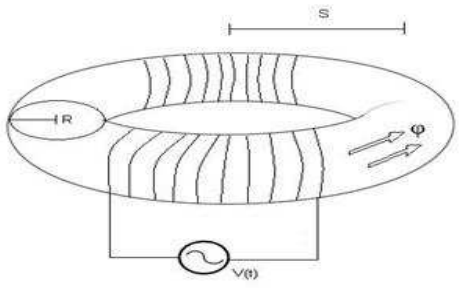

Fig. 1a-Torus physical scheme.

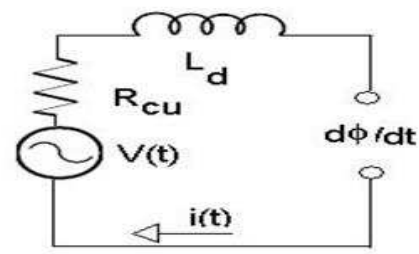

Fig. 1b-Torus electrical scheme
Let's present the dynamical equations set proposed to describe the $\mathrm{MxH}$ relation.

- Magnetic density leakage field

$$
\frac{B(t)}{\mu_{0}}=H(t)+M(t) \quad[\mathrm{A} / \mathrm{m}]
$$

It's the total magnetic field within the material, and $\mu_{0}=4 . \pi \cdot 10^{-7}[T /(A . m)]$.

- $\quad$ Effective intensity magnetic field

$$
H(t)=H_{E}(t)-\tau_{H} \cdot \dot{H}(t)
$$

$\boldsymbol{H}_{\boldsymbol{e}}$ is the applied intensity magnetic field, created by the current passing the coil. The derivative term is due to the Foucault (eddy) macro-current. These currents there exist also in non-magnetic materials, since they are electrical conducting materials.

- Magnetic polarization field

$$
M(t)=f\left(H^{*}\right)-\tau_{M} \cdot \dot{M}(t) \quad[\mathrm{A} / \mathrm{m}]
$$

It's the polarization field created by the material due to the domain wall movement. The derivative term is due to the Foucault (eddy) micro-current, these currents there exist opposing to the domain wall movement. $f$ is an empirical function that fits the anhysterical curve, here we suggest:

$$
\begin{aligned}
& f\left(H^{*}\right)=\frac{\chi}{\mu_{0}} \cdot\left[\beta \cdot \operatorname{atan}\left(\frac{H^{*}}{H_{1}}\right)+(1-\beta) \cdot \operatorname{atan}\left(\frac{H^{*}}{H_{2}}\right)\right] \\
& \text { and also: } \chi \equiv \frac{\mu_{0} \cdot M_{\text {sat }}}{\pi / 2} \text { and } 0 \leq \beta \leq 1
\end{aligned}
$$

The $\boldsymbol{H}_{\boldsymbol{1}}$ and $\boldsymbol{H}_{2}$ parameters describe the magnetization at two different moments: the first one is the domain wall movement and the second one the atoms rotation.

It is convenient to define an auxiliary variable the polarization phase $\theta$ as: $\theta(t) \equiv \mu_{0} \cdot M(t) / \chi$

Rewriting the equations according to the state space description we obtain the variables $\boldsymbol{H}(\boldsymbol{t})$ and $\boldsymbol{\theta}(\boldsymbol{t})$ as a function of the applied field $\boldsymbol{H}_{\boldsymbol{E}}(\boldsymbol{t})$.

$$
\tau_{H} . \dot{H}=H_{E}-H
$$


$\tau_{M} \cdot \dot{\theta}=\beta \cdot \operatorname{atan}\left(\frac{H^{*}}{H_{1}}\right)+(1-\beta) \cdot \operatorname{atan}\left(\frac{H^{*}}{H_{2}}\right)-\theta$

- Liquid intensity magnetic field

$$
H^{*}(t)=H(t)+\alpha \cdot g(\theta)
$$

It is responsible for the induction of the material polarization. The proposal for this field was firstly made by Jiles [2] and differs to the present due to the $\boldsymbol{g}(\boldsymbol{\theta})$ term that represents the Barkhausen noise [3]. An appropriate choice for this function allows us to obtain innumerous distinct equilibrium points. This feature is not present at the linear form of $g(\theta)$.

\subsection{Barkhausen Noise}

The role of $g(\theta)$ function is to induce the Barkhausen noise, and is given by:

$$
g(\theta)=\sin (\sigma . \theta)
$$

One can see that for small values of $\boldsymbol{\sigma}$ this function approaches to a linear, becoming likely the Jiles model. For practice we can define $\boldsymbol{\sigma}$ as:

$\sigma \equiv(4 k+1) \cdot \theta_{\text {sat }} / \theta_{\text {rem }}$; and $\mathrm{k}$ as being an integer number proportional to the domains quantity.

The possible equilibrium points, for null intensity magnetic field, must obey to:

$$
\theta_{\text {equi }}=\beta \cdot \operatorname{atan}\left(\frac{\alpha \cdot \sin \left(\sigma \cdot \theta_{\text {equi }}\right)}{H_{1}}\right)+(1-\beta) \cdot \operatorname{atan}\left(\frac{\alpha \cdot \sin \left(\sigma \cdot \theta_{\text {equi }}\right)}{H_{2}}\right)
$$

The last equilibrium point will be called the material's remnant field; this value allows us to obtain the value for $\boldsymbol{\beta}$ as:

$$
\beta=\frac{\theta_{r e m}-\operatorname{atan}\left(\frac{\alpha}{H_{2}}\right)}{\operatorname{atan}\left(\frac{\alpha}{H_{1}}\right)-\operatorname{atan}\left(\frac{\alpha}{H_{2}}\right)}
$$

A stability study at these points shows an alternation between stable an unstable points. This property implies that there exists a minimum necessary level of magnetization field to jump from one stable point to another neighborhood.

\subsection{Dynamic model parameters}

The parameters set to be adjusted may be divided in pairs according to the physical phenomenon associated with it, so the pair $(\chi, \beta)$ is related to the saturation and remnant magnetic density fields; the pair $\left(H_{1}, H_{2}\right)$ to the an-hysterical function; the pair $(\alpha, \sigma)$ to the Barkhausen noise; and the pair $\left(\tau_{H}, \tau_{M}\right)$ to the Foulcault (eddy) currents. The time constant $\tau_{H}$, due to the macro-currents, depends on the material electrical resistivity and the shelf width of the ferromagnetic core; the time constant $\tau_{M}$, due to the micro-currents, depends also on the material electrical resistivity and the grain size.

Finally the $\boldsymbol{\alpha}, \boldsymbol{\sigma}$ parameters are related to the average magnetic gain created by the domain wall movement that produces the Barkhausen noise. Experiments focused to its detection will present data enough to the necessary parameter fitness [4]. The other parameters have also been discussed.

\section{Parameters adjustment based on hysteresis curves}

Four figure sets are shown, figures 1 to 4 , for four different frequencies: 5 and $50 \mathrm{mHz}$, at $1.5 \mathrm{~T}$ saturation field; and 20 and $60 \mathrm{~Hz}$, at $1.7 \mathrm{~T}$ and $1.5 \mathrm{~T}$ respectively. Each set contains four graphics; the superior graphics present the experimental and theoretical curves for $\mathrm{Bxt}$ and $\mathrm{BxH}$. The inferior graphics present the magnetic energy stocked by the macro and micro currents. These energy were calculated as: $\int H_{E} \cdot \dot{H} \cdot d t$ and $\int H_{E} \cdot \dot{M} \cdot d t$, both added give the total energy $\int H_{E} \cdot \frac{\dot{B}}{\mu_{0}} \cdot d t$. One can observe that the micro-current energy is increasing each cycle, although the macro-current energy is almost constant. The ratio between their amplitudes is about one thousand.

The last graphic is Hxt and $\mathrm{H}_{\mathrm{e}} \mathrm{xt}$, there is no apparent difference between both for low frequencies $(\mathrm{mHz})$ while a small delay can be shown for high frequencies $(60 \mathrm{~Hz})$.

The parameters fitness was obtained searching the minimum squared error between the theoretical en experimental data for the superior figures to all studied frequencies.

Table 1 - Parameters fitness: equations (9) and (10).

\begin{tabular}{|l|l|l|}
\hline$B_{\text {Sat }} \rightarrow \chi$ & {$[\mathrm{T}]$} & 2,1600 \\
\hline$B_{\mathrm{Re} m} \rightarrow \beta$ & {$[\mathrm{T}]$} & 1,0434 \\
\hline$H_{1}$ & {$[\mathrm{~A} / \mathrm{m}]$} & $4.0276 \mathrm{e}+01$ \\
\hline$H_{2}$ & {$[\mathrm{~A} / \mathrm{m}]$} & $1.1452 \mathrm{e}+04$ \\
\hline$\tau_{H}$ & {$[\mathrm{~s}]$} & $2.1088 \mathrm{e}-05$ \\
\hline$\tau_{M}$ & {$[\mathrm{~s}]$} & $1.0544 \mathrm{e}-05$ \\
\hline$\alpha$ & {$[\mathrm{A} / \mathrm{m}]$} & $7.4952 \mathrm{e}+01$ \\
\hline$k \rightarrow \sigma$ & $\#$ & 20 \\
\hline
\end{tabular}

To finalize the analysis two graphics are presented in figure 5, they represent the Barkhouse noise as $\mathrm{dM} / \mathrm{dt} \times \mathrm{t}$ and also $\mathrm{dM} / \mathrm{dt} \times \mathrm{H}$. An histogram was computed, based on this figure, to compare with the Barkhausen noise in [4]. For each $\mathrm{dH}(\mathrm{A} / \mathrm{m})$ interval the edges were counted. The following table was extracted and compered with [4], the similarity gave us the clue to relate the $\mathrm{g}($.) function with the Barkhausen noise. 
Table 2 - Histogram: events number of Barkhausen noise versus Induction magnetic field $\mathrm{H}$.

\begin{tabular}{ccc}
\hline From & Until & $\begin{array}{c}\text { \# of } \\
\text { events }\end{array}$ \\
-250 & -200 & 1 \\
-200 & -150 & 0 \\
-150 & -100 & 1 \\
-100 & -50 & 2 \\
-50 & 0 & 3 \\
0 & 50 & 10 \\
50 & 100 & 21 \\
100 & 150 & 10 \\
150 & 200 & 3 \\
200 & 250 & 2 \\
\hline
\end{tabular}

\section{Conclusion}

The present model was able to represent well the magnetic hysteresis curve from low $(\mathrm{mHz})$ to high $(\mathrm{Hz})$ frequencies. The remnant and saturation field fit well and the number of parameters (6) is not too much when seeing the phenomenon complexity.

A particular feature of this model if compared to others [5], is the Foucault current presence that are entirely responsible for the material losses. Even at low frequencies due to the sinusoidal feed-back term, the system becomes locally unstable and the losses can be explained by this term.

\section{Acknowledgment}

Our sincerely thanks to the researchers of Laboratório de Materiais Magnéticos e Metalrgia do Pó do Instituto de Pesquisas Tecnológicas do Estado de São Paulo, that graceful gave us the necessary data to this work, and a special mention to the drs Julio Carlos Teixeira e Fernado Landgraf.

\section{References}

[1] Pereiralima, P.S., Silva, A.F. , D.L.(2008). Modelo dinâmico para caracterização da curva de histerese magnética: Parte I -Ajuste de parâmetros pela curva an-histerética, Anais do XVII Congresso Brasileiro de Automática CBA set/2008 Juiz de Fora(MG).

[2] Jiles, D.C. e Atherton, D.L.(1983). Ferromagnetic Hysteresis. IEE Transactions on Magnetics. Vol. MAG-19, No5 set/83. DOI: 10.1109/TMAG.1983.1062594

[3] Bertoti, J. H., Fröhlich, K. J. (2001) Elimination of transformer in-rush currents by controlled switching: Part I: treoretical considerations. IEE Transactions on power delivery. Vol 16 no.2 april.

[4] Landgraf, F.J.G. - Microestrutura e histerese magnética nos aços para fins eletromagnéticos. Tese de Livre Docência . Escola Politécnica da Universidade de São Paulo. São Paulo, 2007.

[5] Liorzou, F., Phelps, B., Atherton, D.L. (2000) Macroscopic Models of Magnetization. IEEE transactions on Magnetics. Vol 36 no2 $\operatorname{march} / 2000$. 

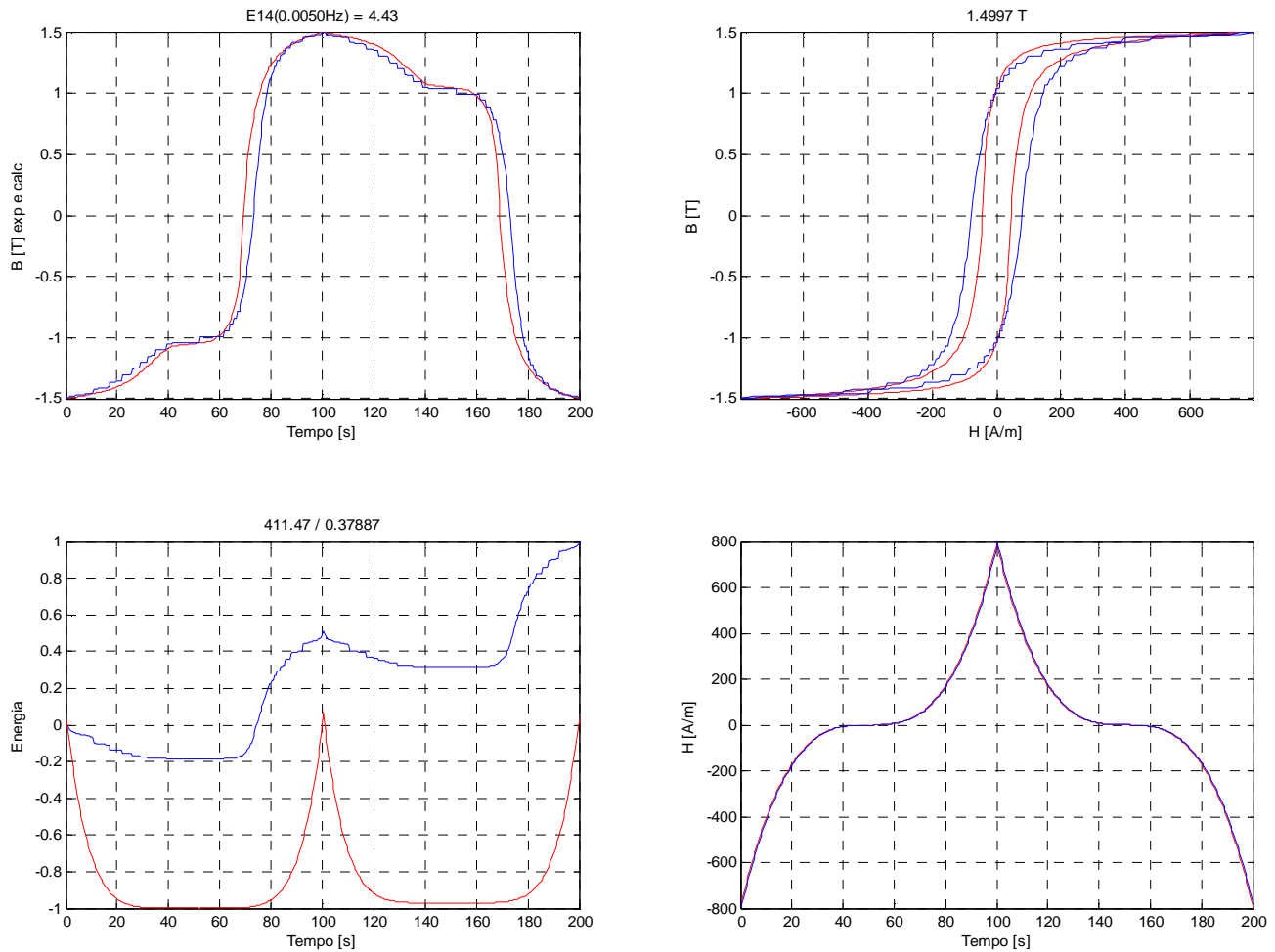

Fig. 1 - Hysteresis curve at $5 \mathrm{mHz}$ and 1,5 T.
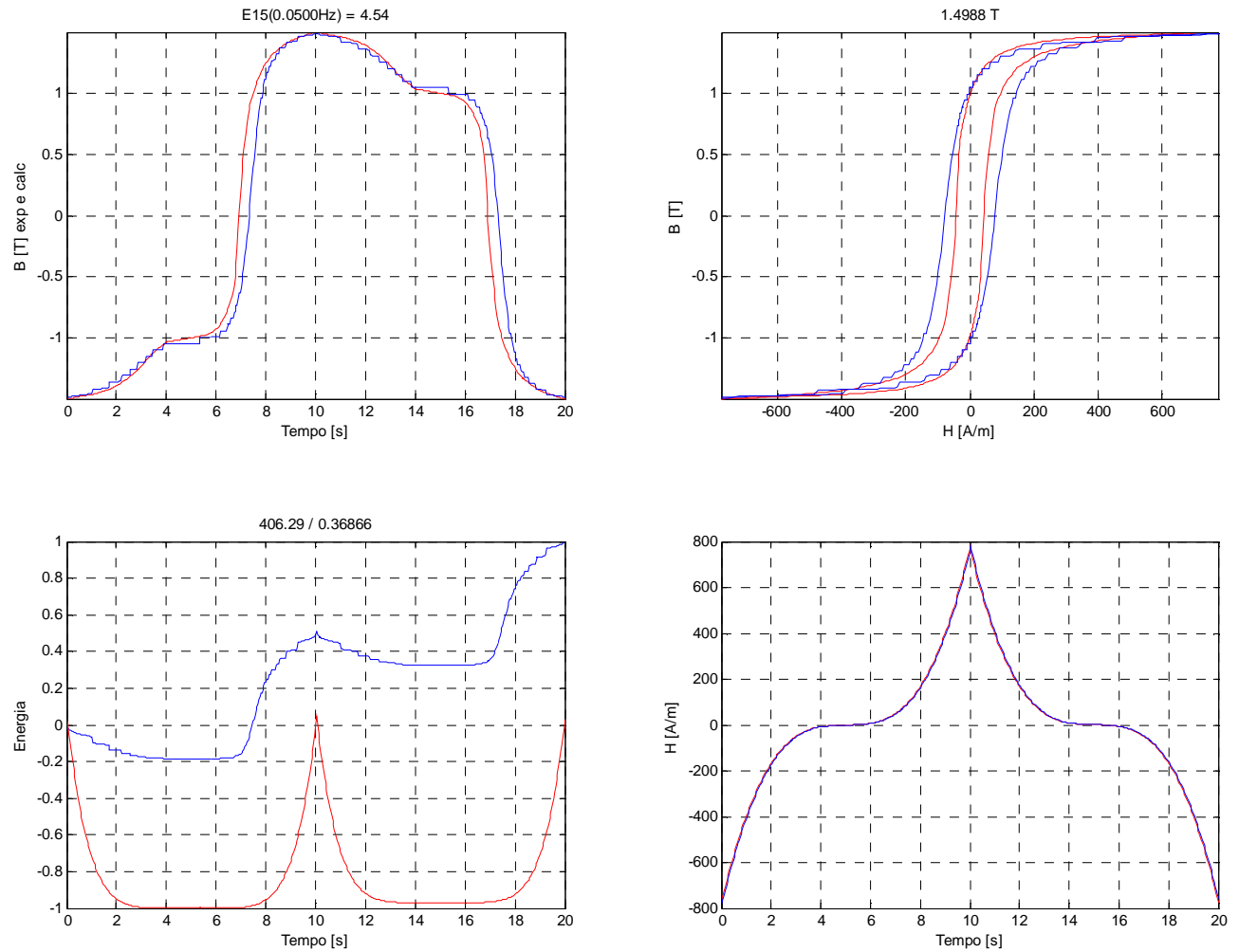

Fig. 2 - Hysteresis curve at $50 \mathrm{mHz}$ and $1,5 \mathrm{~T}$. 

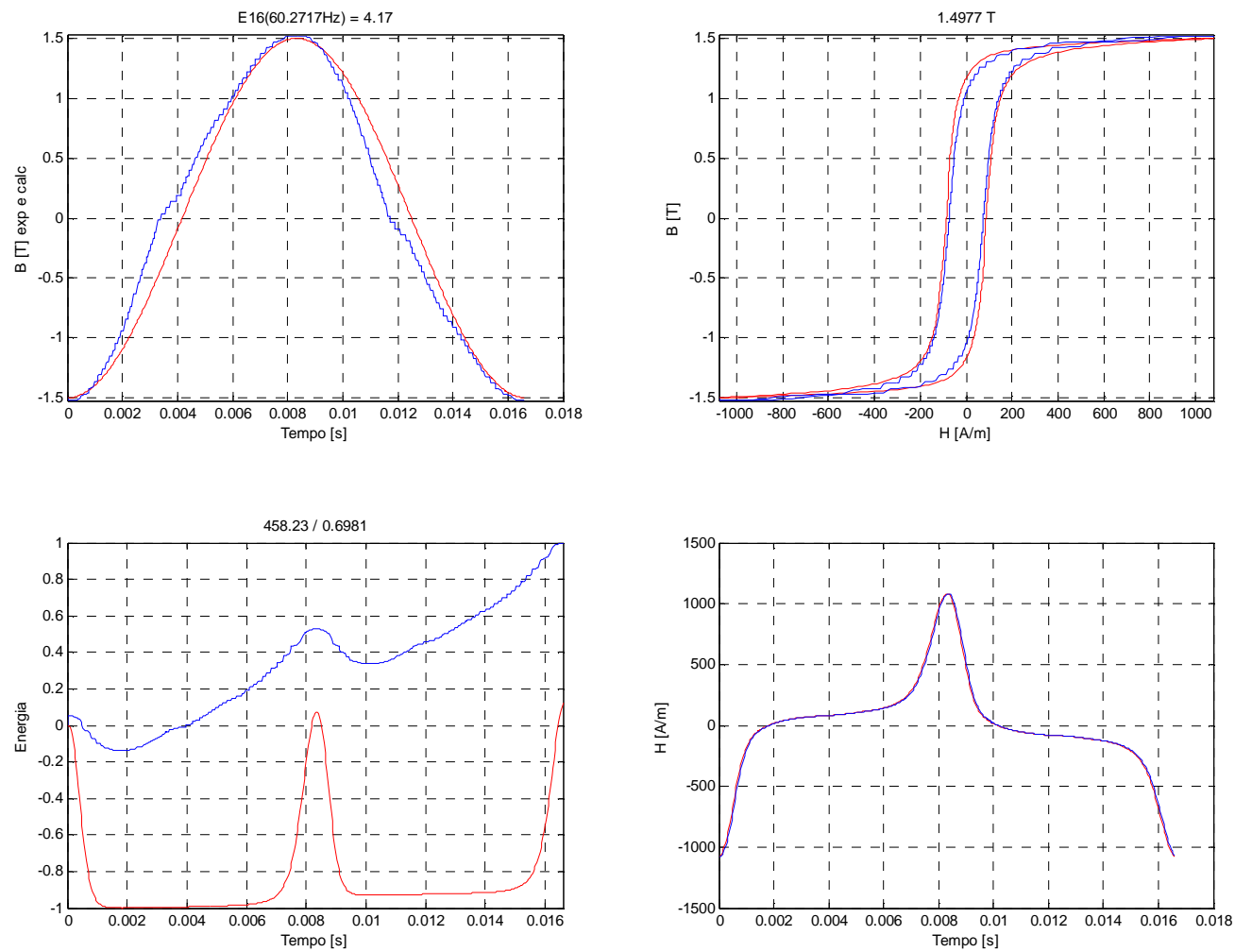

Fig. 3 - Hysteresis curve at $60 \mathrm{~Hz}$ and 1,5 T.
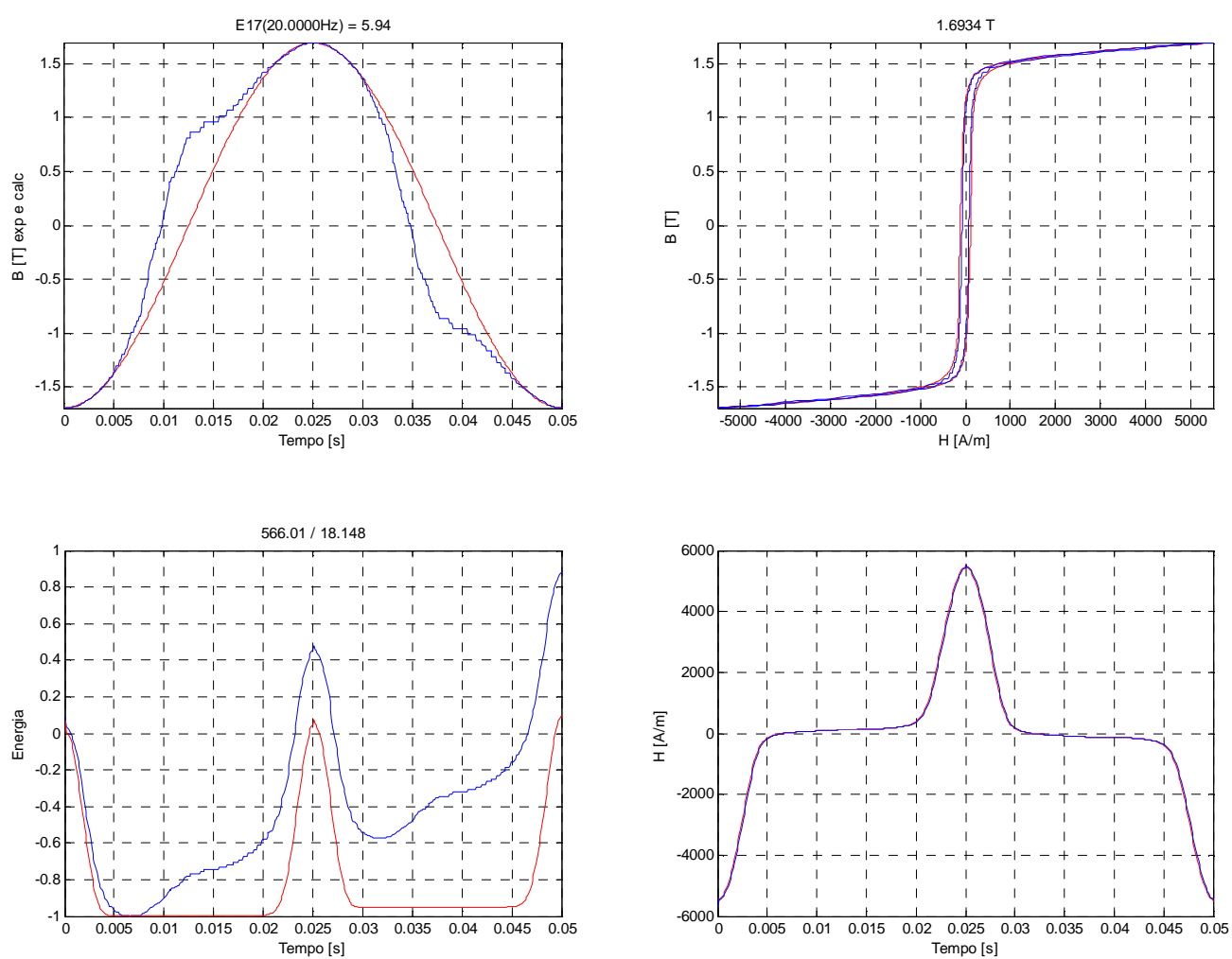

Fig. 4 - Hysteresis curve at $20 \mathrm{~Hz}$ and $1,7 \mathrm{~T}$. 

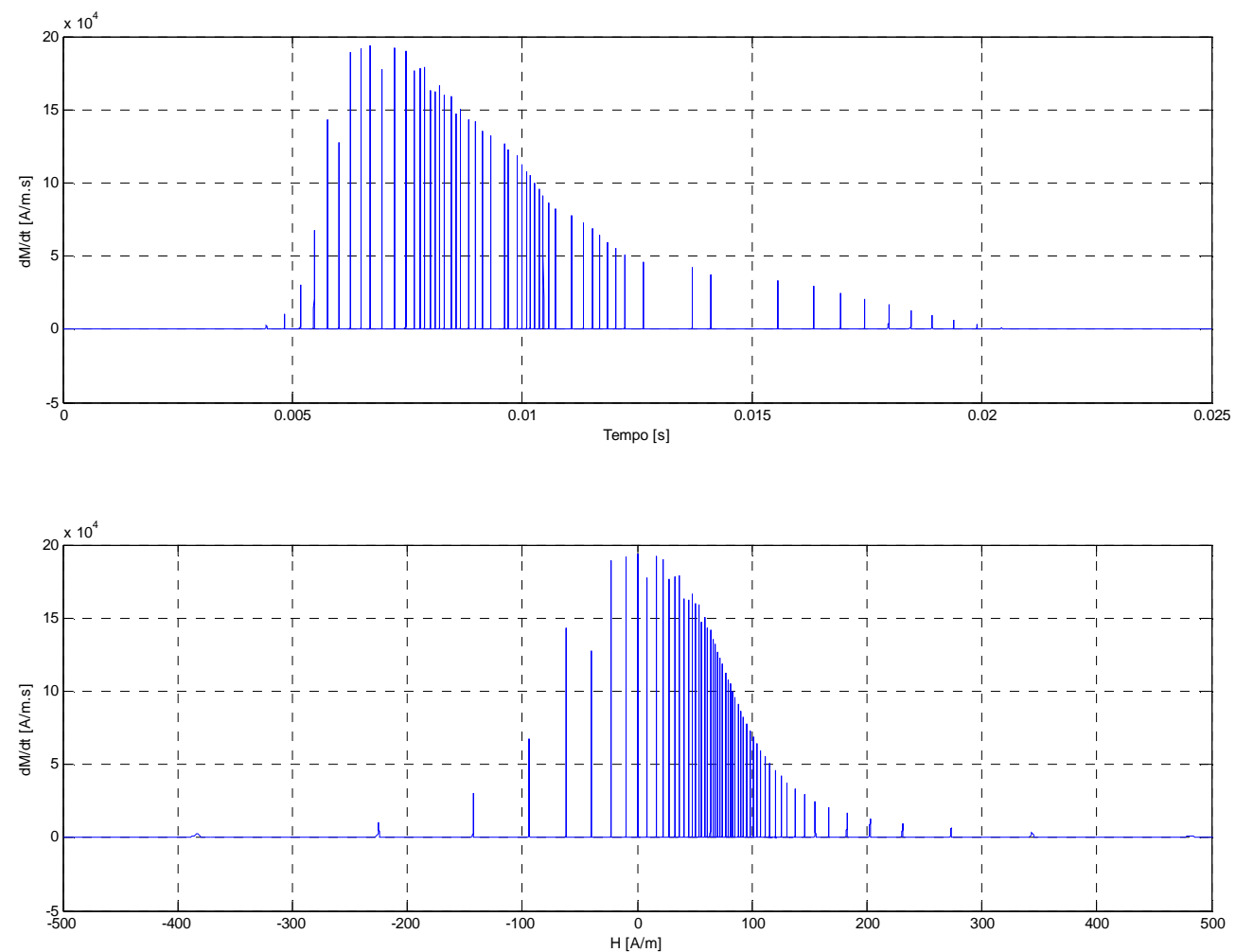

Fig. 5 - Barkhausen noise $(20 \mathrm{~Hz}$ at $1,7 \mathrm{~T})$ 